\title{
Influence of the Center Condition on the Two-Step Secant Method
}

\author{
Abhimanyu Kumar, ${ }^{1}$ D. K. Gupta, ${ }^{1}$ and Shwetabh Srivastava ${ }^{2}$ \\ ${ }^{1}$ Department of Mathematics, Indian Institute of Technology, Kharagpur 721302, India \\ ${ }^{2}$ Department of Mathematics, School of Arts and Sciences, Amrita Vishwa Vidyapeetham (Amrita University), Amritapuri, India
}

Correspondence should be addressed to Shwetabh Srivastava; shwetabhiit@gmail.com

Received 23 June 2017; Accepted 8 August 2017; Published 24 September 2017

Academic Editor: Shamsul Qamar

Copyright ( 2017 Abhimanyu Kumar et al. This is an open access article distributed under the Creative Commons Attribution License, which permits unrestricted use, distribution, and reproduction in any medium, provided the original work is properly cited.

\begin{abstract}
The aim of this paper is to present a new improved semilocal and local convergence analysis for two-step secant method to approximate a locally unique solution of a nonlinear equation in Banach spaces. This study is important because starting points play an important role in the convergence of an iterative method. We have used a combination of Lipschitz and center-Lipschitz conditions on the Fréchet derivative instead of only Lipschitz condition. A comparison is established on different types of center conditions and the influence of our approach is shown through the numerical examples. In comparison to some earlier study, it gives an improved domain of convergence along with the precise error bounds. Finally, some numerical examples including nonlinear elliptic differential equations and integral equations validate the efficacy of our approach.
\end{abstract}

\section{Introduction}

Consider the problem to approximate a locally unique solution $x^{*}$ of

$$
G(x)=0,
$$

where $G: \mathscr{D} \subseteq \mathscr{X} \rightarrow \mathscr{Y}$ is a nonlinear operator. $\mathscr{X}, \mathcal{Y}$ are Banach spaces and $\mathscr{D}$ is an open nonempty convex subset of $\mathscr{X}$. This is one of the very important problems in applied mathematics and engineering science. Many real life problems in diverse areas such as equilibrium theory and elasticity often reduce to solving these equations depending on one or more parameters. Mathematical modeling of many problems uses integral equations, boundary value problems, differential equations, and so forth, whose solutions are obtained by solving scalar equations or a system of equations. Many nonlinear differential equations can be solved by transforming them to matrix equations which give a system of nonlinear equations in $\mathbb{R}^{n}$. Many researchers [1-4] have extensively studied these problems and many methods, both direct and iterative, are developed for their solutions. Good convergence properties, efficiency, and numerical stability are the requirements of all these methods. It is a common problem to choose the good starting points for the iterative methods which ensure the convergence of the iterative method. The semilocal convergence $[2,5,6]$ uses information given at the initial point whereas local convergence $[7,8]$ uses information around the solution. The quadratically convergent Newton's iteration $[9,10]$ is used to solve (1). It is defined for $n \geq 0$ by

$$
x_{n+1}=x_{n}-\Gamma_{n} G\left(x_{n}\right) \text {, }
$$

where $x_{0} \in \mathscr{D}$ is the starting point and $\Gamma_{n}=G^{\prime}\left(x_{n}\right)^{-1} \epsilon$ $L(\mathcal{Y}, \mathscr{X})$ (the set of bounded linear operators from $\mathscr{Y}$ into $\mathscr{X})$. Sufficient conditions for the semilocal convergence with existence ball and error estimates of (2) are given in [11]. The secant iteration $[1,12,13]$ is the simplification of (2) used to solve (1) and is given for $n \geq 0$ by

$$
x_{n+1}=x_{n}-\left[x_{n-1}, x_{n} ; G\right]^{-1} G\left(x_{n}\right) \text {, }
$$

where $x_{-1}, x_{0} \in \mathscr{D}$ are two starting points and $[x, y ; G]$ is the divided difference of order one for $G$ on the points $x, y \in \mathscr{D}$ and satisfies the equality $[x, y ; G](x-y)=G(x)-G(y)$. In 
case of operators, this equality does not hold uniquely unless $\mathscr{X}$ is one-dimensional. In $\mathbb{R}^{n}$, it is defined by a matrix

$$
\begin{array}{r}
{[x, y, G]_{j, k}} \\
=\frac{G_{j}\left(x_{1}, \ldots, x_{k}, y_{k+1}, \ldots, y_{n}\right)-G_{j}\left(x_{1}, \ldots, x_{k-1}, y_{k}, \ldots, y_{n}\right)}{x_{k}-y_{k}}, \\
j, k=1, \ldots, n,
\end{array}
$$

for $x, y \in \mathbb{R}^{n}$ and $G \in L\left(\mathbb{R}^{n}, \mathbb{R}^{n}\right)$. So, many real life problems that require the solution of matrix equations can also be solved by the abovementioned methods.

Recently, an iteration known as the King-Werner iteration originally proposed by King [14] is discussed in [15, 16] along with its local and semilocal convergence using majorizing sequences under the Lipschitz continuous Fréchet derivative of $G$. It is given for $n \geq 1$ by

$$
\begin{aligned}
& x_{n}=x_{n-1}-G^{\prime}\left(\frac{x_{n-1}+y_{n-1}}{2}\right)^{-1} G\left(x_{n-1}\right), \\
& y_{n}=x_{n}-G^{\prime}\left(\frac{x_{n-1}+y_{n-1}}{2}\right)^{-1} G\left(x_{n}\right),
\end{aligned}
$$

where, $x_{0}, y_{0} \in \mathscr{D}$ are the starting iterates. Its order is equal to $1+\sqrt{2}$. A two-step secant iteration with order of convergence same as (5) with its semilocal and local convergence under combination of Lipschitz and center-Lipschitz continuous divided differences of order one using majorizing sequences for solving (1) is described in Banach space setting in [17]. It is defined for $n \geq 0$ by

$$
\begin{aligned}
& x_{n+1}=x_{n}-\left[x_{n}, y_{n} ; G\right]^{-1} G\left(x_{n}\right), \\
& y_{n+1}=x_{n+1}-\left[x_{n}, y_{n} ; G\right]^{-1} G\left(x_{n+1}\right),
\end{aligned}
$$

where $x_{0}, y_{0} \in \mathscr{D}$ are starting iterates.

In this paper, iteration (6) is considered for solving (1) along with its semilocal and local convergence analysis under weaker Lipschitz continuity condition on divided differences of order one on the involved operator $G$ in Banach space setting. The influence on the domain by our approach is shown by some numerical examples. It provides the improved error estimations along with the better information on the location of solutions. Semilocal convergence of (6) is studied, which improves the applicability of the method corresponding to some earlier study $[17,18]$. It is shown by our work that earlier studies for (6) do not hold while the new convergence criteria hold. For local convergence analysis, weaker center-Lipschitz continuity condition is used in place of a combination of Lipschitz and center-Lipschitz continuity conditions. Larger convergence ball is obtained through this study in comparison to the older one.

The paper is arranged as follows. Introduction forms Section 1. In Section 2, the semilocal convergence analysis of (6) under weaker convergence conditions on divided differences of operator $G$ is established. In Section 3, local convergence analysis of (6) is established using only centerLipschitz continuity condition on divided differences. In Section 4, numerical examples are given to validate the theoretical results obtained by us. Finally, conclusions and references are included in Section 5.

\section{Semilocal Convergence}

In this section, firstly, we provide a lemma that will be used to provide the semilocal convergence theorem of (6).

Lemma 1. Let $k_{0}, k, k_{1}, k_{2}, \eta$, and s be nonnegative parameters and $\alpha$ be the unique root of the polynomial defined by

$$
g(t)=k t^{3}+k_{0} t^{2}+\left(k_{1}+k_{2}\right) t-\left(k_{1}+k_{2}\right)
$$

and sequences $\left\{t_{n}\right\}$ and $\left\{s_{n}\right\}$ defined for $n \geq 0$ by $t_{0}=0, t_{1}=\eta$, $s_{0}=s$,

$$
\begin{aligned}
& s_{1}=t_{1}+\frac{k_{0} t_{1}+k s_{0}}{1-k s_{0}} t_{1}, \\
& t_{2}=t_{1}+\frac{k_{0} t_{1}+k s_{0}}{1-\left(k_{0} t_{1}+k s_{1}\right)} t_{1},
\end{aligned}
$$

and for all $n \geq 1$ by

$$
\begin{aligned}
& s_{n+1}=t_{n+1}+\frac{k_{1}\left(t_{n+1}-t_{n}\right)+k_{2}\left(s_{n}-t_{n}\right)}{1-\left(k_{0} t_{n}+k s_{n}\right)}\left(t_{n+1}-t_{n}\right), \\
& t_{n+2}=t_{n+1}+\frac{k_{1}\left(t_{n+1}-t_{n}\right)+k_{2}\left(s_{n}-t_{n}\right)}{1-\left(k_{0} t_{n+1}+k s_{n+1}\right)}\left(t_{n+1}-t_{n}\right) .
\end{aligned}
$$

Supposing

$$
0<\frac{k_{0} t_{1}+k s_{0}}{1-\left(k_{0} t_{1}+k s_{1}\right)} \leq \alpha \leq 1-\left(k_{0}+k\right) t_{1},
$$

then sequences $\left\{s_{n}\right\},\left\{t_{n}\right\}$ are well defined, increasing, and bounded above by $t^{* *}=t_{1} /(1-\alpha)$ and converge to their least upper bound $t^{*}$ which satisfies $t_{1} \leq t^{*} \leq t^{* *}$. Moreover, the following estimates hold for all $n \geq 1$ :

$$
\begin{aligned}
& 0 \leq s_{n+1}-t_{n+1} \leq \alpha\left(t_{n+1}-t_{n}\right), \\
& 0 \leq t_{n+2}-t_{n+1} \leq \alpha\left(t_{n+1}-t_{n}\right) .
\end{aligned}
$$

Proof. From (7), $g(0)=-\left(k_{1}+k_{2}\right)$ and $g(1)=\left(k+k_{0}\right)$. Using intermediate value theorem, $g$ has at least one root in $(0,1)$; also, it is increasing in this interval. So, it has a unique root in this interval which is denoted by $\alpha$. Suppose $t_{1}=0$; then, all terms of sequences $\left\{t_{n}\right\}$ and $\left\{s_{n}\right\}$ will be equal to 0 and Lemma 1 holds in this case. Taking $t_{1}=\eta>0$, then (11) is true if

$$
\begin{aligned}
& 0<\frac{k_{1}\left(t_{n+1}-t_{n}\right)+k_{2}\left(s_{n}-t_{n}\right)}{1-\left(k_{0} t_{n}+k s_{n}\right)} \leq \alpha, \\
& 0<\frac{k_{1}\left(t_{n+1}-t_{n}\right)+k_{2}\left(s_{n}-t_{n}\right)}{1-\left(k_{0} t_{n+1}+k s_{n+1}\right)} \leq \alpha,
\end{aligned}
$$

$$
t_{n} \leq s_{n}
$$

for each $n=1,2, \ldots$. 
This implies that $0 \leq s_{n}-t_{n} \leq \alpha^{n}\left(t_{1}-t_{0}\right)$ and $0 \leq t_{n+1}-t_{n} \leq$ $\alpha^{n}\left(t_{1}-t_{0}\right)$. Now, instead of showing (12), it will be sufficient to show that

$$
\begin{gathered}
\left(k_{1}+k_{2}\right) t_{1} \alpha^{n-1}+\frac{k_{0} t_{1}}{1-\alpha}\left(1-\alpha^{n+1}\right) \\
+\frac{k t_{1}}{1-\alpha}\left(1-\alpha^{n+2}\right) \leq 1 .
\end{gathered}
$$

From (13), we are motivated to construct a recurrent polynomial

$$
\begin{aligned}
f_{n}(t)= & \left(k_{1}+k_{2}\right) t_{1} t^{n-1}+\frac{k_{0} t_{1}}{1-t}\left(1-t^{n+1}\right) \\
& +\frac{k t_{1}}{1-t}\left(1-t^{n+2}\right)-1 .
\end{aligned}
$$

Replacing $n$ by $n+1$ in (14), this gives

$$
\begin{aligned}
f_{n+1}(t)= & \left(k_{1}+k_{2}\right) t_{1} t^{n}+\frac{k_{0} t_{1}}{1-t}\left(1-t^{n+2}\right) \\
& +\frac{k t_{1}}{1-t}\left(1-t^{n+3}\right)-1 .
\end{aligned}
$$

Now, from (7), (14), and (15) and the help of some algebraic manipulations, we have

$$
f_{n+1}(t)=f_{n}(t)+g(t) t^{n-1} t_{1} .
$$

Using (16), we get $f_{n+1}(\alpha)=f_{n}(\alpha)$; also, $f_{n}(t)$ is an increasing function in $(0,1)$. Let us define a function $R(t)$ on $(0,1)$ by

$$
R(t)=\lim _{n \rightarrow \infty} f_{n}(t) .
$$

Now, we need to show only $R(\alpha) \leq 0$. Using (10), this assertion can be proved easily and, thus, Lemma 1 is established.

Next, we provide a semilocal convergence theorem followed by Lemma 1 for (6).

Theorem 2. Let $G: \mathscr{D} \subseteq \mathscr{X} \rightarrow \mathscr{Y}$ be a nonlinear operator; $k_{0}, k, k_{1}, k_{2}, \eta$, and $s$ are given parameters. Denote $A_{n}=\left[x_{n}, y_{n} ; G\right]$ for $n \geq 0$. Under the hypothesis of Lemma 1, the following assumptions hold in $\overline{\mathscr{B}\left(x_{0}, t^{*}\right)} \subseteq \mathscr{D}$ :

$$
\begin{array}{ll}
\left(A_{1}\right) & A_{0}^{-1} \in L(Y, X) \\
\left(A_{2}\right) & \left\|A_{0}^{-1} G\left(x_{0}\right)\right\| \leq \eta \\
\left(A_{3}\right) & \left\|x_{0}-y_{0}\right\| \leq s \\
\left(A_{4}\right) & \left\|G^{\prime}\left(x_{0}\right)^{-1}\left([x, y ; G]-G^{\prime}\left(x_{0}\right)\right)\right\| \\
& \leq k_{0}\left\|x-x_{0}\right\|+k\left\|y-x_{0}\right\| \\
\left(A_{5}\right) & \left\|G^{\prime}\left(x_{0}\right)^{-1}\left([x, y ; G]-G^{\prime}(z)\right)\right\| \\
& \leq k_{1}\|x-z\|+k_{2}\|y-z\|
\end{array}
$$

Staring with suitable $x_{0}, y_{0} \in \overline{\mathscr{B}\left(x_{0}, t^{*}\right)}$, sequences $\left\{x_{n}\right\}$ and $\left\{y_{n}\right\}$ defined in (6) are well defined, remain in $\overline{\mathscr{B}\left(x_{0}, t^{*}\right)}$, and converge to a solution $x^{*}$ in $\overline{\mathscr{B}\left(x_{0}, t^{*}\right)}$ of (1). Moreover, the following estimates hold for each $n \geq 0$ :

$$
\begin{aligned}
\left\|y_{n}-x_{n}\right\| & \leq s_{n}-t_{n}, \\
\left\|x_{n+1}-x_{n}\right\| & \leq t_{n+1}-t_{n}, \\
\left\|x^{*}-x_{n}\right\| & \leq t^{*}-t_{n} .
\end{aligned}
$$

Further, if there exists $R>t^{*}$ such that $\mathscr{B}\left(x_{0}, R\right) \subseteq \mathscr{D}$ and $k_{0} t^{*}+k R<1$, then $x^{*}$ is the only solution of (1) in $\mathscr{B}\left(x_{0}, R\right) \cap \mathscr{D}$.

Proof. Using mathematical induction on $n$, we shall show that (19) hold true. For $n=1$, this follows directly from (18) which shows that $x_{1} \in \overline{\mathscr{B}\left(x_{0}, t^{*}\right)}$. Using Banach lemma and (18), we get $\left\|A_{0}^{-1} G^{\prime}\left(x_{0}\right)\right\| \leq 1 /(1-k s)$. Next,

$$
\begin{aligned}
& \left\|y_{1}-x_{1}\right\|=\left\|A_{0}^{-1}\left(G\left(x_{1}\right)-G\left(x_{0}\right)+G\left(x_{0}\right)\right)\right\| \\
& \quad \leq\left\|A_{0}^{-1} G^{\prime}\left(x_{0}\right)\right\| \\
& \cdot\left(\left\|G^{\prime}\left(x_{0}\right)^{-1}\left(\left[x_{1}, x_{0} ; G\right]-G^{\prime}\left(x_{0}\right)\right)\right\|\right. \\
& \left.+\left\|G^{\prime}\left(x_{0}\right)^{-1}\left(\left[x_{0}, y_{0} ; G\right]-G^{\prime}\left(x_{0}\right)\right)\right\|\right)\left\|x_{1}-x_{0}\right\| \\
& \quad \leq \frac{k_{0}\left\|x_{1}-x_{0}\right\|+k\left\|x_{0}-y_{0}\right\|}{1-k s}\left\|x_{1}-x_{0}\right\| \\
& \leq \frac{k_{0} t_{1}+k s}{1-k s} t_{1}=s_{1}-t_{1}, \\
& \left\|y_{1}-x_{0}\right\| \leq\left\|y_{1}-x_{1}\right\|+\left\|x_{1}-x_{0}\right\| \leq s_{1}-t_{1}+t_{1}-t_{0} \\
& =s_{1}<t^{*} .
\end{aligned}
$$

This shows that $y_{1} \in \overline{\mathscr{B}\left(x_{0}, t^{*}\right)}$

$$
\begin{aligned}
\left\|I-G^{\prime}\left(x_{0}\right)^{-1} A_{1}\right\| & =\left\|G^{\prime}\left(x_{0}\right)^{-1}\left(A_{1}-G^{\prime}\left(x_{0}\right)\right)\right\| \\
& \leq k_{0}\left\|x_{1}-x_{0}\right\|+k\left\|y_{1}-x_{0}\right\| \\
& \leq k_{0} t_{1}+k s_{1}<1 .
\end{aligned}
$$

Using Banach lemma [4] on invertible operators, we get

$$
\left\|A_{1}^{-1} G^{\prime}\left(x_{0}\right)\right\| \leq \frac{1}{1-\left(k_{0} t_{1}+k s_{1}\right)} .
$$

Now,

$$
\begin{aligned}
\left\|x_{2}-x_{1}\right\| & \leq\left\|A_{1}^{-1} G\left(x_{1}\right)\right\| \\
& \leq\left\|A_{1}^{-1} G^{\prime}\left(x_{0}\right)\right\|\left\|G^{\prime}\left(x_{0}\right)^{-1} G\left(x_{1}\right)\right\| \\
& \leq \frac{k_{0} t_{1}+k s}{1-\left(k_{0} t_{1}+k s_{1}\right)} t_{1}=t_{2}-t_{1}, \\
\left\|x_{2}-x_{0}\right\| & \leq\left\|x_{2}-x_{1}\right\|+\left\|x_{1}-x_{0}\right\| \leq t_{2}-t_{1}+t_{1}-t_{0} \\
& =t_{2}<t^{*} .
\end{aligned}
$$


This implies $x_{2} \in \overline{\mathscr{B}\left(x_{0}, t^{*}\right)}$ and thus (19) is true for $n=1$. Now, from (6),

$$
\begin{aligned}
\left\|y_{2}-x_{2}\right\| & \leq\left\|A_{1}^{-1} G\left(x_{2}\right)\right\| \\
& \leq\left\|A_{1}^{-1} G^{\prime}\left(x_{0}\right)\right\|\left\|G^{\prime}\left(x_{0}\right)^{-1} G\left(x_{2}\right)\right\| \\
& \leq \frac{k_{1}\left(t_{2}-t_{1}\right)+k_{2}\left(s_{1}-t_{1}\right)}{1-\left(k_{0} t_{1}+k s_{1}\right)}\left(t_{2}-t_{1}\right) \\
& =s_{2}-t_{2}, \\
\left\|y_{2}-x_{0}\right\| & \leq\left\|y_{2}-x_{2}\right\|+\left\|x_{2}-x_{0}\right\| \leq s_{2}-t_{2}+t_{2}-t_{0} \\
& =s_{2}<t^{*} .
\end{aligned}
$$

This shows that $x_{2}, y_{2} \in \overline{\mathscr{B}\left(x_{0}, t^{*}\right)}$. Thus, replacing $x_{1}, y_{1}, x_{2}, y_{2}$ by $x_{n+1}, y_{n+1}, x_{n+2}, y_{n+2}$ and proceeding in a similar manner, this gives the notion that $\left\{x_{n}\right\}$ is a complete sequence in Banach space $\mathscr{X}$ such that it converges to some $x^{*} \in \overline{\mathscr{B}\left(x_{0}, t^{*}\right)}$. Now, to show that $x^{*}$ is a solution of $(1)$,

$$
\begin{aligned}
& \left\|G^{\prime}\left(x_{0}\right)^{-1} G\left(x_{n+1}\right)\right\| \\
& \quad \leq\left(k_{1}\left(t_{n+1}-t_{n}\right)+k_{2}\left(s_{n}-t_{n}\right)\right) t_{n+1}-t_{n} \longrightarrow 0
\end{aligned}
$$

as $n \longrightarrow \infty$.

So, $G\left(x^{*}\right)=0$. Suppose $y^{*}$ is another solution of (1) such that $G\left(y^{*}\right)=0$. Let $T=\left[x^{*}, y^{*} ; G\right]$ be an operator and

$$
\begin{aligned}
& \left\|G^{\prime}\left(x_{0}\right)^{-1}\left(G^{\prime}\left(x_{0}\right)-T\right)\right\| \\
& \quad \leq k_{0}\left\|x^{*}-x_{0}\right\|+k\left\|y^{*}-x_{0}\right\| \leq k_{0} t^{*}+k R^{*}<1 .
\end{aligned}
$$

It follows that $x^{*}=y^{*}$ and this establishes Theorem 2 .

To make the paper self-content, we present the lemma and semilocal convergence theorem of (6) that can be found in [18].

Lemma 3 (see [18]). Let $\widetilde{k_{0}}, \widetilde{k}, \widetilde{k_{1}}, \widetilde{k_{2}}, \eta$, and $s$ be nonnegative parameters and $\alpha$ be the unique root of the polynomial defined by

$$
g(t)=\widetilde{k} t^{3}+\widetilde{k_{0}} t^{2}+\left(\widetilde{k_{1}}+\widetilde{k_{2}}\right) t-\left(\widetilde{k_{1}}+\widetilde{k_{2}}\right)
$$

and sequences $\left\{l_{n}\right\}$ and $\left\{r_{n}\right\}$ defined for $n \geq 0$, by $l_{0}=0, l_{1}=$ $\eta, r_{0}=s$

$$
\begin{aligned}
& r_{1}=l_{1}+\left(\widetilde{k_{0}} l_{1}+\widetilde{k} r_{0}\right) l_{1}, \\
& l_{2}=l_{1}+\frac{\widetilde{k_{0}} l_{1}+\widetilde{k} r_{0}}{1-\left(\widetilde{k_{0}} l_{1}+\widetilde{k} r_{1}\right)} l_{1},
\end{aligned}
$$

and for all $n \geq 1$ by

$$
\begin{aligned}
& r_{n+1}=l_{n+1}+\frac{\widetilde{k_{1}}\left(l_{n+1}-l_{n}\right)+\widetilde{k_{2}}\left(r_{n}-l_{n}\right)}{1-\left(k_{0} l_{n}+k r_{n}\right)}\left(l_{n+1}-l_{n}\right), \\
& l_{n+2}=l_{n+1}+\frac{\widetilde{k_{1}}\left(l_{n+1}-l_{n}\right)+\widetilde{k_{2}}\left(r_{n}-l_{n}\right)}{1-\left(\widetilde{k_{0}} l_{n+1}+\widetilde{k}\left(r_{n+1}+r_{0}\right)\right)}\left(l_{n+1}-l_{n}\right) .
\end{aligned}
$$

Supposing

$$
\begin{aligned}
& \frac{\widetilde{k_{0}}\left(t_{1}-t_{0}\right)+\widetilde{k} s_{0}}{1-\left(\widetilde{k_{0}}\left(t_{1}-t_{0}\right)+\widetilde{k}\left(s_{1}+s_{0}\right)\right)} \leq \alpha, \\
& 0<\frac{\widetilde{k_{0}} l_{1}}{1-\alpha}+\frac{\widetilde{k} l_{1}}{1-\alpha}+\widetilde{k} r_{0}<1,
\end{aligned}
$$

then sequences $\left\{r_{n}\right\},\left\{l_{n}\right\}$ are well defined, increasing, and bounded above by $l^{* *}=l_{1} /(1-\alpha)$ and converge to their least upper bound $l^{*}$ which satisfies $l_{1} \leq l^{*} \leq l^{* *}$. Moreover, the following estimates hold for all $n \geq 1$ :

$$
\begin{aligned}
& 0 \leq r_{n+1}-l_{n+1} \leq \alpha\left(l_{n+1}-l_{n}\right), \\
& 0 \leq l_{n+2}-l_{n+1} \leq \alpha\left(l_{n+1}-l_{n}\right), \\
& l_{n} \leq r_{n} .
\end{aligned}
$$

Theorem 4 (see [18]). Let $G: \mathscr{D} \subseteq \mathscr{X} \rightarrow \mathscr{Y}$ be a nonlinear operator; $k_{0}, k, k_{1}, k_{2}, \eta$, and $s$ are given parameters. Denote $A_{n}=\left[x_{n}, y_{n} ; G\right]$ for $n \geq 0$. Under the hypothesis of Lemma 3, the following assumptions hold in $\overline{\mathscr{B}\left(x_{0}, l^{*}\right)} \subseteq \mathscr{D}$ :

$$
\begin{array}{ll}
\left(A_{1}\right) & A_{0}^{-1} \in L(Y, X) \\
\left(A_{2}\right) \quad & \left\|A_{0}^{-1} G\left(x_{0}\right)\right\| \leq \eta \\
\left(A_{3}\right) & \left\|x_{0}-y_{0}\right\| \leq s \\
\left(A_{4}\right) & \left\|A_{0}^{-1}\left([x, y ; G]-\left[x_{0}, y_{0} ; G\right]\right)\right\| \\
& \leq \widetilde{k_{0}}\left\|x-x_{0}\right\|+\widetilde{k}\left\|y-y_{0}\right\| \\
\left(A_{5}\right) \quad\left\|A_{0}^{-1}([x, y ; G]-[u, v ; G])\right\| \\
\quad \leq \widetilde{k_{1}}\|x-u\|+\widetilde{k_{2}}\|y-v\|
\end{array}
$$

for all $x, y, u, v \in \mathscr{D}$.

Starting with suitable $x_{0}, y_{0} \in \overline{\mathscr{B}\left(x_{0}, l^{*}\right)}$, sequences $\left\{x_{n}\right\}$ and $\left\{y_{n}\right\}$ defined in (6) are well defined, remain in $\overline{\mathscr{B}}\left(x_{0}, l^{*}\right)$, and converge to a unique solution $x^{*}$ in $\overline{\mathscr{B}}\left(x_{0}, l^{*}\right)$ of (1). Moreover, the following estimates hold for each $n \geq 0$ :

$$
\begin{aligned}
\left\|y_{n}-x_{n}\right\| & \leq r_{n}-l_{n}, \\
\left\|x_{n+1}-x_{n}\right\| & \leq l_{n+1}-l_{n}, \\
\left\|x^{*}-x_{n}\right\| & \leq l^{*}-t_{n} .
\end{aligned}
$$

Further, if there exists $\widetilde{R}>l^{*}$ such that $\mathscr{B}\left(x_{0}, \widetilde{R}\right) \subseteq \mathscr{D}$ and $\widetilde{k_{0}} l^{*}+\widetilde{k} \widetilde{R}<1$, then $x^{*}$ is the only solution of $(1)$ in $\mathscr{B}\left(x_{0}, \widetilde{R}\right) \cap \mathscr{D}$.

Previous assertions [17] are made for iteration (6) as follows:

$$
\begin{aligned}
& \left\|A_{0}^{-1}\left([x, y ; G]-A_{0}\right)\right\| \leq K\left(\left\|x-x_{0}\right\|+\left\|y-y_{0}\right\|\right), \\
& \left\|A_{0}^{-1}([x, y ; G]-[u, v ; G])\right\| \\
& \quad \leq K_{1}(\|x-u\|+\|y-v\|) .
\end{aligned}
$$


One can easily see that our conditions are more general than (35), and with conditions (35), the following majorizing sequences are obtained:

$$
\begin{aligned}
p_{0}= & 0, \\
p_{1}= & \eta, \\
q_{0}= & s, \\
q_{1}= & p_{1}+K_{1}\left(p_{1}+q_{0}\right) p_{1}, \quad \text { for } n \geq 1, \\
p_{n+1}= & p_{n} \\
& +\frac{K_{1}\left(p_{n}-p_{n-1}+q_{n-1}-p_{n-1}\right)}{1-K_{0}\left(p_{n}-p_{0}+q_{n}+q_{0}\right)}\left(p_{n}-p_{n-1}\right), \\
q_{n+1}= & p_{n+1} \\
& +\frac{K_{1}\left(p_{n+1}-p_{n}+q_{n}-p_{n}\right)}{1-K_{0}\left(p_{n}-p_{0}+q_{n}+q_{0}\right)}\left(p_{n+1}-p_{n}\right) .
\end{aligned}
$$

\section{Local Convergence}

In this section, the local convergence of (6) to solve (1) is established under center-Lipschitz condition on divided differences of order one of the involved operator $G$.

Theorem 5. Let $l_{0}, l \geq 0$ be given parameters and $G: \mathscr{D} \times \mathscr{D} \rightarrow$ $y$ be a nonlinear divided difference operator such that for all $x, y \in \mathscr{D}$

$$
\begin{aligned}
& G\left(x^{*}\right)=0, \\
& G^{\prime}\left(x^{*}\right)^{-1} \in L(\mathcal{Y}, x), \\
& \left\|G^{\prime}\left(x^{*}\right)^{-1}\left([x, y ; G]-G^{\prime}\left(x^{*}\right)\right)\right\| \\
& \quad \leq l_{0}\left\|x-x^{*}\right\|+l\left\|y-x^{*}\right\|,
\end{aligned}
$$

and $\overline{\mathscr{B}\left(x^{*}, r^{*}\right)} \subseteq \mathscr{D}$, where $r^{*}=1 /\left(2 l+3 l_{0}\right)$. Then, sequences $\left\{x_{n}\right\},\left\{y_{n}\right\}$ of (6) starting from $x_{0}, y_{0} \in \mathscr{B}\left(x^{*}, r^{*}\right)$ are well defined, remain in $\overline{\mathscr{B}\left(x^{*}, r^{*}\right)}$ for each $n=0,1,2, \ldots$, and converge to $x^{*}$. Moreover, the following error estimates hold:

$$
\begin{aligned}
& \left\|x_{n+1}-x^{*}\right\| \\
& \quad \leq \frac{\left(2 l_{0}\left\|x_{n}-x^{*}\right\|+l\left\|y_{n}-x^{*}\right\|\right)}{1-\left(l_{0}\left\|x_{n}-x^{*}\right\|+l\left\|y_{n}-x^{*}\right\|\right)}\left\|x_{n}-x^{*}\right\| \\
& \quad<\left\|x_{n}-x^{*}\right\|<r^{*} .
\end{aligned}
$$

Additionally, if there exists $R^{*}>r^{*}$ such that $\overline{\mathscr{B}\left(x^{*}, R^{*}\right)} \subseteq \mathscr{D}$ and $R^{*}<1 / l$, then $x^{*}$ is the only solution of $(1)$ in $\overline{\mathscr{B}\left(x^{*}, R^{*}\right)} \cap$ D.

Proof. For $x_{0}, y_{0} \in \mathscr{B}\left(x^{*}, r^{*}\right)$ and using (37), we get

$$
\begin{aligned}
\left\|I-G^{\prime}\left(x^{*}\right)^{-1} A_{0}\right\| & =\left\|G^{\prime}\left(x^{*}\right)^{-1}\left(G^{\prime}\left(x^{*}\right)-A_{0}\right)\right\| \\
& \leq l_{0}\left\|x_{0}-x^{*}\right\|+l\left\|y_{0}-x^{*}\right\| \\
& <\left(l_{0}+l\right) r^{*}<1 .
\end{aligned}
$$

So, by Banach lemma, $A_{0}^{-1}$ exists and

$$
\left\|A_{0}^{-1} G^{\prime}\left(x^{*}\right)\right\| \leq \frac{1}{1-\left(l_{0}+l\right) /\left(2 l+3 l_{0}\right)}=\frac{2 l+3 l_{0}}{l+2 l_{0}} .
$$

Using (37) and (40) and hypothesis of Theorem 5, we have

$$
\begin{aligned}
& \left\|x_{1}-x^{*}\right\|=\left\|x_{0}-x^{*}-A_{0}^{-1}\left(G\left(x_{0}\right)-G\left(x^{*}\right)\right)\right\| \\
& \leq\left\|A_{0}^{-1} G^{\prime}\left(x^{*}\right)\right\| \\
& \cdot\left(\left\|G^{\prime}\left(x^{*}\right)^{-1}\left(\left[x_{0}, y_{0} ; G\right]-G^{\prime}\left(x^{*}\right)\right)\right\|\right. \\
& \left.+\left\|G^{\prime}\left(x^{*}\right)^{-1}\left(\left[x_{0}, x^{*} ; G\right]-G^{\prime}\left(x^{*}\right)\right)\right\|\right)\left\|x_{0}-x^{*}\right\| \\
& \leq \frac{\left(2 l_{0}\left\|x_{0}-x^{*}\right\|+l\left\|y_{0}-x^{*}\right\|\right)}{1-\left(l_{0}\left\|x_{0}-x^{*}\right\|+l\left\|y_{0}-x^{*}\right\|\right)}\left\|x_{0}-x^{*}\right\| \\
& <\frac{\left(2 l_{0}+l\right) r^{*}}{1-\left(l_{0}+l\right) r^{*}}\left\|x_{0}-x^{*}\right\|=\left\|x_{0}-x^{*}\right\|<r^{*} .
\end{aligned}
$$

Again, using (37)-(41) and hypothesis of Theorem 5, we get

$$
\begin{aligned}
& \left\|y_{1}-x^{*}\right\|=\left\|x_{1}-x^{*}-A_{0}^{-1}\left(G\left(x_{1}\right)-G\left(x^{*}\right)\right)\right\| \\
& \leq\left\|A_{0}^{-1} G^{\prime}\left(x^{*}\right)\right\| \\
& \cdot\left(\left\|G^{\prime}\left(x^{*}\right)^{-1}\left(\left[x_{0}, y_{0} ; G\right]-G^{\prime}\left(x^{*}\right)\right)\right\|\right. \\
& \left.+\left\|G^{\prime}\left(x^{*}\right)^{-1}\left(\left[x_{1}, x^{*} ; G\right]-G^{\prime}\left(x^{*}\right)\right)\right\|\right)\left\|x_{1}-x^{*}\right\| \\
& \leq \frac{\left(l_{0}\left(\left\|x_{0}-x^{*}\right\|+\left\|x_{1}-x^{*}\right\|\right)+l\left\|y_{0}-x^{*}\right\|\right)}{1-\left(l_{0}\left\|x_{0}-x^{*}\right\|+l\left\|y_{0}-x^{*}\right\|\right)} \| x_{1} \\
& -x^{*}\left\|<\frac{\left(2 l_{0}+l\right) r^{*}}{1-\left(l_{0}+l\right) r^{*}}\right\| x_{1}-x^{*}\|=\| x_{1}-x^{*} \| .
\end{aligned}
$$

This shows that $x_{1}, y_{1} \in \mathscr{B}\left(x^{*}, r^{*}\right)$. Clearly, using induction on $n$,

$$
\begin{aligned}
& \left\|x_{n+1}-x^{*}\right\|=\left\|x_{n}-x^{*}-A_{n}^{-1}\left(G\left(x_{n}\right)-G\left(x^{*}\right)\right)\right\| \\
& \quad \leq\left\|A_{n}^{-1} G^{\prime}\left(x^{*}\right)\right\| \\
& \cdot\left(\left\|G^{\prime}\left(x^{*}\right)^{-1}\left(\left[x_{n}, y_{n} ; G\right]-G^{\prime}\left(x^{*}\right)\right)\right\|\right. \\
& \left.\quad+\left\|G^{\prime}\left(x^{*}\right)^{-1}\left(\left[x_{n}, x^{*} ; G\right]-G^{\prime}\left(x^{*}\right)\right)\right\|\right)\left\|x_{n}-x^{*}\right\| \\
& \quad \leq \frac{\left(2 l_{0}\left\|x_{n}-x^{*}\right\|+l\left\|y_{n}-x^{*}\right\|\right)}{1-\left(l_{0}\left\|x_{n}-x^{*}\right\|+l\left\|y_{n}-x^{*}\right\|\right)}\left\|x_{n}-x^{*}\right\| \\
& \quad<\left\|x_{n}-x^{*}\right\|<r^{*} .
\end{aligned}
$$

This shows (38). Now, let $y^{*}$ be another solution of (1) in $\mathscr{B}\left(x^{*}, R^{*}\right)$ such that $G\left(y^{*}\right)=0$. Using (37), this gives

$$
\begin{aligned}
& \left\|G^{\prime}\left(x^{*}\right)^{-1}\left(\left[x^{*}, y^{*} ; G\right]-G^{\prime}\left(x^{*}\right)\right)\right\| \leq l\left\|y^{*}-x^{*}\right\| \\
& \quad<1 .
\end{aligned}
$$

This shows that $x^{*}$ is the unique solution of (1) in $\overline{\mathscr{B}\left(x^{*}, R^{*}\right)} \cap$ D. 




FIGURE 1: Domain of (1).

\section{Numerical Examples}

In this section, some numerical examples are given to show the effectiveness of the present study. We have used approach-1, approach-2, and approach-3 by (18), (33), and (35), respectively.

Example 1. Let $\mathscr{X}=\mathscr{Y}=\mathscr{R}, \mathscr{D}=\mathscr{B}\left(x_{0}, 1\right)$ and define function $G$ on $\mathscr{D}$ by

$$
G(x)=x^{3}-\theta
$$

We take $x_{0}=1$ and $y_{0}$ free in order to find a relation between $\theta$ and $y_{0}$ for which all the criteria for ensuring the convergence are satisfied. In Figure 1, we have taken horizontal axis for $y_{0}$ and vertical axis for $\theta$. With the help of (6), we obtain $s=\left|1-y_{0}\right|, \eta=|1-\theta| /\left(1+y_{0}+y_{0}^{2}\right)$, $k_{0}=2, k=1, k_{1}=8 / 3, k_{2}=4 / 3, \widetilde{k_{0}}=6, \widetilde{k}=3, \widetilde{k_{1}}=4$, $K_{1}=\widetilde{k_{2}}=8$, and $K=4$. The efficacy of our approach can be seen in Figure 1.

For comparing the error estimation where all approaches including the older approach are satisfied, we take the domain $\mathscr{D}=\mathscr{B}\left(x_{0}, 0.3\right)$ and fix $\theta=0.75, y_{0}=0.95$. In this case, we get

$$
G(x)=x^{3}-0.75
$$

and $s=0.05, \eta=0.0876424, k_{0}=1.53333, k=0.766666$, $k_{1}=1.73333, k_{2}=0.86666, \widetilde{k}=0.8063, K=\widetilde{k_{0}}=1.6126$, $\widetilde{k_{1}}=0.9115$, and $K_{1}=\widetilde{k_{2}}=1.8230$. Comparison of error estimations with different approaches is given in Table 1.

From Figure 1, it can be seen that there exists some combination of $y_{0}$ and $\theta$ where the condition used earlier fails. When all conditions hold, then it gives the precise error bounds. Thus, the claim made by us in the abstract and the Introduction is justified here.

Example 2. Let $\mathscr{X}=\mathscr{Y}=\mathscr{C}[0,1]$, the space of all continuous functions defined in $[0,1]$ equipped with the max-norm. Let $\mathscr{D}=\{x \in \mathscr{C}[0,1] ;\|x\| \leq R\}$ such that $R=1$ and define $G$ on $\mathscr{D}$ by

$$
\begin{aligned}
G(x)=x(s)-f(s)-\frac{1}{8} \int_{0}^{1} G_{1}(s, t) x(t)^{3} d t & \\
& x \in \mathscr{C}[0,1], s \in[0,1],
\end{aligned}
$$

where $f \in \mathscr{C}[0,1]$ is a given function and the kernel $G_{1}$ is Green's function:

$$
G_{1}(s, t)= \begin{cases}t(1-s), & \text { if } t \leq s \\ s(1-t), & \text { if } s \leq t\end{cases}
$$

Now, one can represent a linear operator $G^{\prime}(x)$ by

$$
\begin{aligned}
&\left(G^{\prime}(x)\right) v(s)=v(s)-\frac{3}{8} \int_{0}^{1} G_{1}(s, t) x(t)^{2} v(t) d t, \\
& v \in \mathscr{C}[0,1], s \in[0,1] .
\end{aligned}
$$

Choose $x_{0}(s)=f(s)=s$ and $y_{0}(s)=2 s$; we obtain

$$
\left\|G\left(x_{0}\right)\right\| \leq \frac{1}{64} \text {. }
$$

It can be easily seen that

$$
\begin{aligned}
& \left\|[x, y ; G]-G^{\prime}(z)\right\| \\
& \quad \leq \int_{0}^{1}\left\|G^{\prime}(\gamma x+(1-\gamma) y)-G^{\prime}(z)\right\| d \gamma \\
& \quad \leq \frac{1}{64}\left(\left\|x^{2}-z^{2}\right\|+\left\|y^{2}-z^{2}\right\|+\left\|x y-z^{2}\right\|\right) .
\end{aligned}
$$

$\eta=0.1404, k_{0}=0.0984, k=0.0492, k_{1}=0.1311, k_{2}=$ $0.0565, \widetilde{k}=0.0526, K=\widetilde{k_{0}}=0.1228, \widetilde{k_{1}}=0.1404, K_{1}=\widetilde{k_{2}}=$ 0.0702 , and $s=1$. Comparison of the error estimation with the older one is given in Table 2 .

Example 3. Consider the partial differential equation

$$
\triangle u=u^{3}
$$

where

$$
\Delta=\frac{\partial^{2}}{\partial \xi_{1}^{2}}+\frac{\partial^{2}}{\partial \xi_{2}^{2}}
$$

is the two-dimensional Laplace operator. These types of equations arise in the theory of gas dynamics [19]. Assume that (52) is satisfied in the rectangular domain $\mathscr{D}=\left\{\left(\xi_{1}, \xi_{2}\right) \in\right.$ $\left.\mathscr{R}^{2} ; 0 \leq \xi_{1} \leq 1,0 \leq \xi_{2} \leq 1\right\}$ with the Dirichlet boundary condition given by

$$
\begin{aligned}
& u\left(\xi_{1}, 0\right)=2 \xi_{1}^{2}-\xi_{1}+1, \quad u\left(\xi_{1}, 1\right)=2, \\
& u\left(0, \xi_{2}\right)=2 \xi_{2}^{2}-\xi_{2}+1, \quad u\left(1, \xi_{2}\right)=2 .
\end{aligned}
$$


TABLE 1: Comparison of error estimation for (46).

\begin{tabular}{|c|c|c|c|c|c|}
\hline$t_{n+1}-t_{n}$ & $s_{n}-t_{n}$ & $l_{n+1}-l_{n}$ & $r_{n}-l_{n}$ & $p_{n+1}-p_{n}$ & $q_{n}-p_{n}$ \\
\hline $1.9250 e-02$ & $1.5700 e-02$ & $2.1668 e-02$ & $1.5922 e-02$ & $3.2362 e-02$ & $2.1993 e-02$ \\
\hline $1.2013 e-03$ & $1.1508 e-03$ & $1.6866 e-03$ & $1.5928 e-03$ & $6.1234 e-03$ & $5.3343 e-03$ \\
\hline $4.9236 e-06$ & $4.9113 e-06$ & $1.1047 e-05$ & $1.1002 e-05$ & $2.4979 e-04$ & $2.4428 e-04$ \\
\hline $8.3814 e-11$ & $8.3813 e-11$ & $4.8224 e-10$ & $4.8222 e-10$ & $4.3972 e-07$ & $4.3937 e-07$ \\
\hline 0 & 0 & 0 & 0 & $1.3772 e-12$ & $1.3772 e-12$ \\
\hline
\end{tabular}

TABLE 2: Comparison of error estimation for Example 2.

\begin{tabular}{|c|c|c|c|c|c|}
\hline$t_{n+1}-t_{n}$ & $s_{n}-t_{n}$ & $l_{n+1}-l_{n}$ & $r_{n}-l_{n}$ & $p_{n+1}-p_{n}$ & $q_{n}-p_{n}$ \\
\hline $9.3052 e-03$ & $9.0388 e-03$ & $1.0231 e-02$ & $9.4607 e-03$ & $1.9542 e-02$ & $2.2479 e-02$ \\
\hline $1.6579 e-05$ & $1.6594 e-05$ & $2.3267 e-05$ & $2.3239 e-05$ & $1.3390 e-04$ & $1.3363 e-04$ \\
\hline $5.5371 e-11$ & $5.5371 e-11$ & $1.2339 e-10$ & $1.2339 e-10$ & $5.8417 e-09$ & $5.8416 e-09$ \\
\hline
\end{tabular}

In order to transform (52) into a system of nonlinear equations, central divided differences scheme has been used. This leads to a nonlinear system of equations:

$$
\begin{aligned}
u_{i+1, j}-4 u_{i, j}+u_{i, j+1}+u_{i, j-1}- & h^{2} u_{i, j}=0, \\
& i=1, \ldots, n, j=1, \ldots, m .
\end{aligned}
$$

$u(i, j)$ denotes the estimation $u\left(\xi_{1, i}, \xi_{2, j}\right)$. Now, taking $n=$ $m=5$, this generates a $6 \times 6$ mesh. Boundary values can be obtained from (54), and to find interior points, we transform interior values as $x_{1}=u_{1,1}, x_{2}=u_{2,1}, x_{3}=u_{3,1}, x_{4}=$ $u_{4,1}, x_{5}=u_{1,2}, x_{6}=u_{2,2}, x_{7}=u_{3,2}, x_{8}=u_{4,2}, x_{9}=u_{1,3}, x_{10}=$ $u_{2,3}, x_{11}=u_{3,3}, x_{12}=u_{4,3}, x_{13}=u_{1,4}, x_{14}=u_{2,4}, x_{15}=$ $u_{3,4}, x_{16}=u_{4,4}$. So, for $\mathscr{X}=\mathscr{Y}=\mathscr{R}^{16}$, the system can be expressed as

$$
G(x)=A x+h^{2} \phi(x)-b=0,
$$

where $A, h$, and $b$ are given by

$$
\begin{aligned}
& h=\frac{1}{5}, \\
& A=\left(\begin{array}{llll}
B & C & 0 & 0 \\
C & B & C & 0 \\
0 & C & B & C \\
0 & 0 & C & B
\end{array}\right), \\
& B=\left(\begin{array}{cccc}
4 & -1 & 0 & 0 \\
-1 & 4 & -1 & 0 \\
0 & -1 & 4 & -1 \\
0 & 0 & -1 & 4
\end{array}\right), \\
& C=-I_{4}, \quad 10 \\
& \phi(t)=\left(x_{1}^{3}, x_{2}^{3}, \ldots, x_{16}^{3}\right)^{T}, \\
& b=\left(\frac{44}{25}, \frac{23}{25}, \frac{28}{25}, \frac{87}{25}, \frac{23}{25}, 0,0,2, \frac{28}{25}, 0,0,2, \frac{87}{25}, 2,2,4\right)^{T} .
\end{aligned}
$$

Now,

$$
\begin{aligned}
& \left\|G^{\prime}(x)-G^{\prime}(y)\right\|=\| \operatorname{diag}\left\{3 h^{2}\right. \\
& \left.\cdot \max _{0 \leq x_{i}, y_{i} \leq 1}\left\{x_{1}^{2}-y_{1}^{2}, x_{2}^{2}-y_{2}^{2}, \ldots, x_{16}^{2}-y_{16}^{2}\right\}\right\} \| \\
& \leq 3 h^{2} \max _{1 \leq i \leq 16}\left\|x_{i}^{2}-y_{i}^{2}\right\| \leq 3 h^{2}\left\|x^{2}-y^{2}\right\| .
\end{aligned}
$$

So,

$$
\begin{aligned}
& \left\|[x, y ; G]-G^{\prime}(z)\right\| \\
& \quad \leq \int_{0}^{1}\left\|G^{\prime}(\gamma x+(1-\gamma) y)-G^{\prime}(z)\right\| d \gamma \\
& \quad \leq h^{2}(\|x+z\|\|x-z\|+\|y+x+z\|\|y-z\|) .
\end{aligned}
$$

We choose $x_{0}=(4 / 5,4 / 5, \ldots, 4 / 5)^{T}$ and $y_{0}(i)=x_{0}(i)+0.1$, and we get $k_{0}=0.2169, k=0.1084, k_{1}=0.2168, k_{2}=0.1205$, $s=0.1, \eta=1.0425, \widetilde{k}=0.1070813, K=\widetilde{k_{0}}=0.2201, \widetilde{k_{1}}=$ 0.2379 , and $K_{1}=\widetilde{k_{2}}=0.1189$. In this example, approach-3 does not hold but approach-1 and approach-2 hold well. Now, we compare approach-1 and approach- 2 for this example and a comparison is given in Table 3. Next, we use (6) to solve (56) and the approximate solution is given with stopping criterion $\left\|x_{n}-x_{n-1}\right\| \leq 10^{-15}$. The approximate solution is then given in Table 4.

Interpolating the value of Table 4 , we get the numerical approximation of the solution which can be seen in Figure 2 .

Example 4. Let $\mathscr{X}=\mathscr{Y}=R^{3}, \mathscr{D}=R^{3}$, and $x^{*}=(0,0,0)^{T}$ and define a function $G$ on $\mathscr{D}$ by

$$
G(x, y, z)=\left(e^{x}-1, y^{2}+y, z\right)^{T} .
$$

For $t=(x, y, z)^{T}$,

$$
G^{\prime}(t)=\left(\begin{array}{ccc}
e^{x} & 0 & 0 \\
0 & 2 y+1 & 0 \\
0 & 0 & 1
\end{array}\right)
$$


TABLE 3: Comparison of error estimation for Example 3.

\begin{tabular}{lccc}
\hline$t_{n+1}-t_{n}$ & $s_{n}-t_{n}$ & $l_{n+1}-l_{n}$ & $r_{n}-l_{n}$ \\
\hline 0.3897 & 0.2497 & 0.4029 & 0.2504 \\
$9.1893 e-02$ & $7.6239 e-02$ & $9.9774 e-02$ & $8.1506 e-02$ \\
$5.7163 e-03$ & $5.4747 e-03$ & $6.9079 e-03$ & $6.5728 e-03$ \\
$2.3179 e-05$ & $2.3121 e-05$ & $3.4807 e-05$ & $3.4695 e-05$ \\
$3.8625 e-10$ & $3.8624 e-10$ & $8.9732 e-10$ & $8.9730 e-10$ \\
\hline
\end{tabular}

TABLE 4: Approximate solution of (52).

\begin{tabular}{lc}
\hline$i$ & $x_{i}^{*}$ \\
\hline 1 & 0.967514648571165 \\
2 & 1.073142808305482 \\
3 & 1.255308661675940 \\
4 & 1.547504427760980 \\
5 & 1.073142808305482 \\
6 & 1.199182696602124 \\
7 & 1.359712017969179 \\
8 & 1.602945733655613 \\
9 & 1.255308661675940 \\
10 & 1.359712017969179 \\
11 & 1.481965315289151 \\
12 & 1.669313085344323 \\
13 & 1.547504427760980 \\
14 & 1.602945733655613 \\
15 & 1.669313085344323 \\
16 & 1.778410018624668 \\
\hline
\end{tabular}

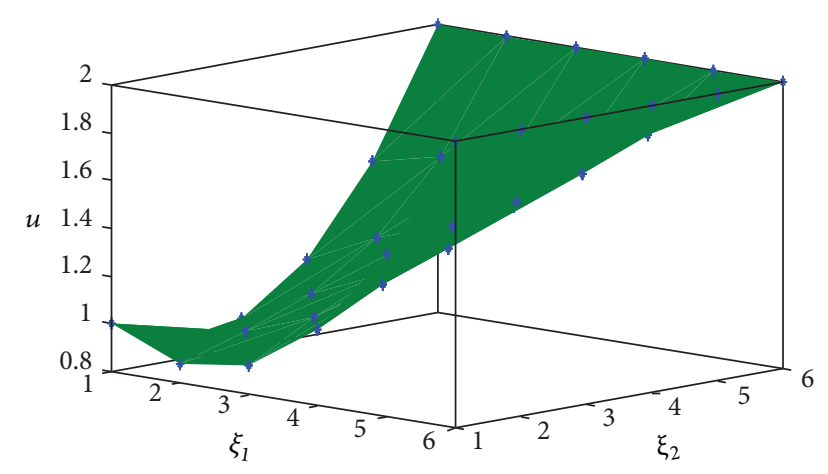

FIgURE 2: Approximated solution of (52).

Using max-norm of rows, one can easily find that $G^{\prime}\left(x^{*}\right)=$ $\operatorname{diag}\{1,1,1\}, l_{0}=e / 2$, and $l=1, r^{*}=0.164543$. So, from Theorem 5, (6) starting from $x_{0}, y_{0} \in \mathscr{B}\left(x^{*}, r^{*}\right)$ converges to $x^{*}$.

For comparison of the radius of ball convergence with a previous study, we take another example which satisfies the previous condition given in [17].
Example 5. Let $\mathscr{X}=\mathscr{Y}=R, \mathscr{D}=R, x^{*}=0$ and define a function $G$ on $\mathscr{D}$ by

$$
G(x)=e^{x}-1 .
$$

Clearly, $G^{\prime}\left(x^{*}\right)=1$. In this case, we get $l_{0}=l=(e-1) / 2$, and using Theorem 5, we get $r^{*}=0.23279$ which is bigger than the corresponding radius 0.20002 in [17].

Example 6. Let $\mathscr{X}=\mathscr{Y}=\mathscr{C}[0,1]$, the space of continuous functions defined on $[0,1]$, equipped with the max-norm and $\mathscr{D}=\overline{\mathscr{B}(0,1)}$. Define $G$ on $\mathscr{D}$, given by

$$
G(x)(s)=x(s)-5 \int_{0}^{1} s t x^{3}(t) d t
$$

$s \in[0,1]$. We obtain $l_{0}=l=3.75$, and using this value, we find that $r^{*}=0.05333$ which is bigger than the corresponding radius 0.039052 in [17].

\section{Conclusions}

In this work, the semilocal and local convergence analysis for two-step secant method is established. A comparison is established on different types of center conditions used earlier for the convergence analysis. It is shown that the approach used in this paper gives precise error bounds along with the better information to the solution. It is also shown that sometimes earlier condition fails to converge but the sufficient conditions used in this paper hold. Moreover, it gives precise error bounds. Finally, some numerical examples including gas dynamics and integral equations validate the theoretical results obtained in this study.

\section{Conflicts of Interest}

The authors declare that they have no conflicts of interest.

\section{References}

[1] A. Margeñán and I. K. Argyros, "New improved convergence analysis for the secant method," Mathematics and Computers in Simulation, vol. 119, pp. 161-170, 2016.

[2] S. Singh, D. K. Gupta, E. Martínez, and J. L. Hueso, "Semilocal and local convergence of a fifth order iteration with Fréchet derivative satisfying Hölder condition," Applied Mathematics and Computation, vol. 276, pp. 266-277, 2016.

[3] P. K. Parida and D. K. Gupta, "Recurrence relations for semilocal convergence of a Newton-like method in Banach spaces," 
Journal of Mathematical Analysis and Applications, vol. 345, no. 1, pp. 350-361, 2008.

[4] J. M. Ortega and W. C. Rheinboldt, Iterative Solution of Nonlinear Equations in Several Variables, Academic Press, New York, NY, USA, 1970.

[5] I. K. Argyros, A. Cordero, A. Margeñán, and J. R. Torregrosa, "On the convergence of a damped Newton-like method with modified right hand side vector," Applied Mathematics and Computation, vol. 266, Article ID 21280, pp. 927-936, 2015.

[6] P. Maroju, R. Behl, and S. S. Motsa, "Convergence of a parameter based iterative method for solving nonlinear equations in Banach spaces," in S.S. Convergence of a parameter based iterative method for solving nonlinear equations in Banach spaces, pp. 10-1007, II. Ser, Rend. Circ. Mat. Palermo, 2016.

[7] I. K. Argyros and S. George, "Local convergence of deformed Halley method in Banach space under HOLder continuity conditions," Journal of Nonlinear Science and its Applications. JNSA, vol. 8, no. 3, pp. 246-254, 2015.

[8] S. Amat, S. Busquier, and J. M. Gutiérrez, "On the local convergence of secant-type methods," International Journal of Computer Mathematics, vol. 81, no. 9, pp. 1153-1161, 2004.

[9] J. A. Ezquerro and M. Á. Hernández-Verón, “On the accessibility of Newton's method under a Hölder condition on the first derivative," Algorithms (Basel), vol. 8, no. 3, pp. 514-528, 2015.

[10] J. A. Ezquerro and M. Á. Hernández-Verón, "Enlarging the domain of starting points for Newton's method under center conditions on the first Fréchet-derivative," Journal of Complexity, vol. 33, pp. 89-106, 2016.

[11] L. V. Kantorovich and G. P. Akilov, Functional Analysis, Pergamon Press, Nauka, 1982.

[12] A. Margeñán and I. K. Argyros, "New semilocal and local convergence analysis for the secant method," Applied Mathematics and Computation, vol. 262, pp. 298-307, 2015.

[13] I. K. Argyros and A. Margeñán, "Expanding the applicability of the secant method under weaker conditions," Applied Mathematics and Computation, vol. 266, Article ID 21308, pp. 10001012, 2015.

[14] R. F. King, "Tangent methods for nonlinear equations," Numerische Mathematik, vol. 18, pp. 298-304, 1971/72.

[15] I. K. Argyros and H. Ren, "On the convergence of efficient King-Werner-type methods of order $1+2$," Journal of Computational and Applied Mathematics, vol. 285, pp. 169-180, 2015.

[16] W. Werner, "Some supplementary results on the $1+\sqrt{ } 2$ order method for the solution of nonlinear equations," Numerische Mathematik, vol. 38, no. 3, pp. 383-392, 1981/82.

[17] H. Ren and I. K. Argyros, "On the convergence of King-Wernertype methods of order $1+\sqrt{ } 2$ free of derivatives," Applied Mathematics and Computation, vol. 256, pp. 148-159, 2015.

[18] A. Kumar, D. Gupta, E. Martínez, and S. Singh, "Semilocal convergence of a Secant-type method under weak Lipschitz conditions in Banach spaces," Journal of Computational and Applied Mathematics, 2017.

[19] L. B. Rall, Computational Solution of Nonlinear Operator Equations, With an appendix by Ramon E. Moore, John Wiley \& Sons, Inc, New York, NY, USA, 1969. 




Advances in

Operations Research

vatersals

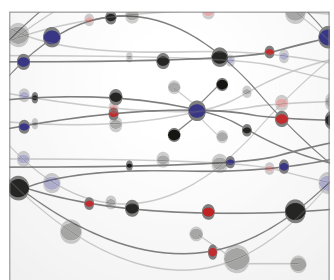

\section{The Scientific} World Journal
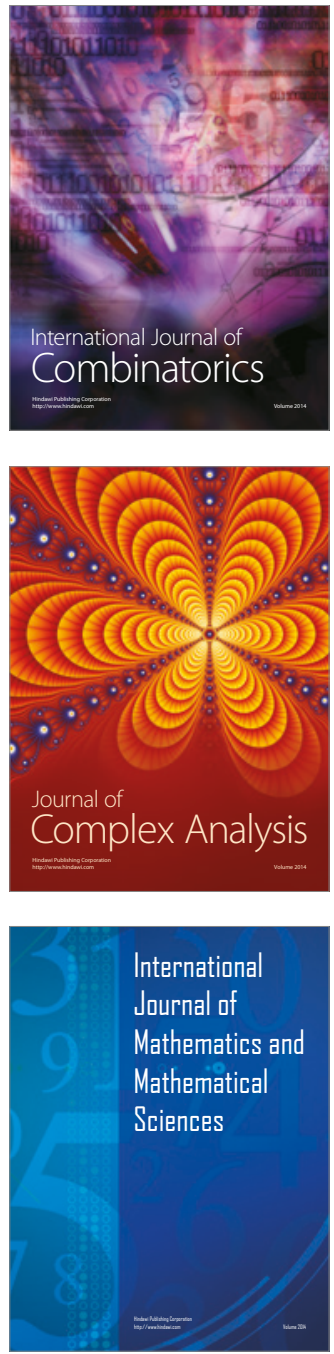
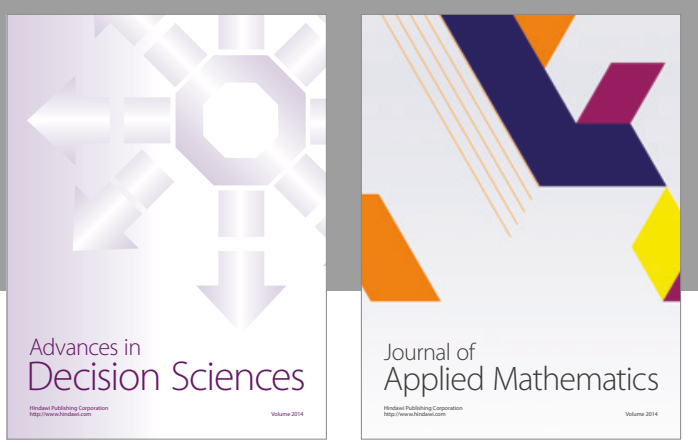

Algebra

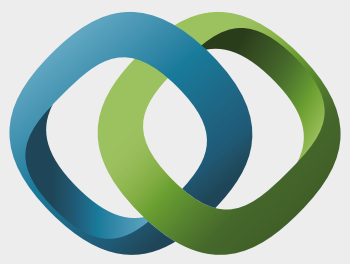

\section{Hindawi}

Submit your manuscripts at

https://www.hindawi.com
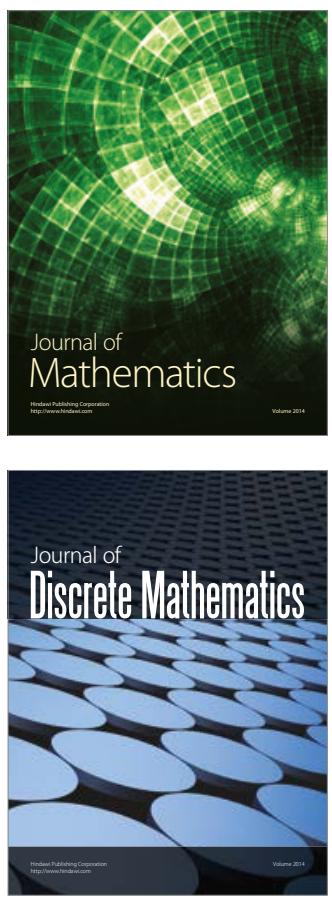

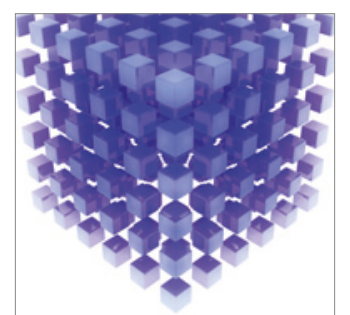

Mathematical Problems in Engineering
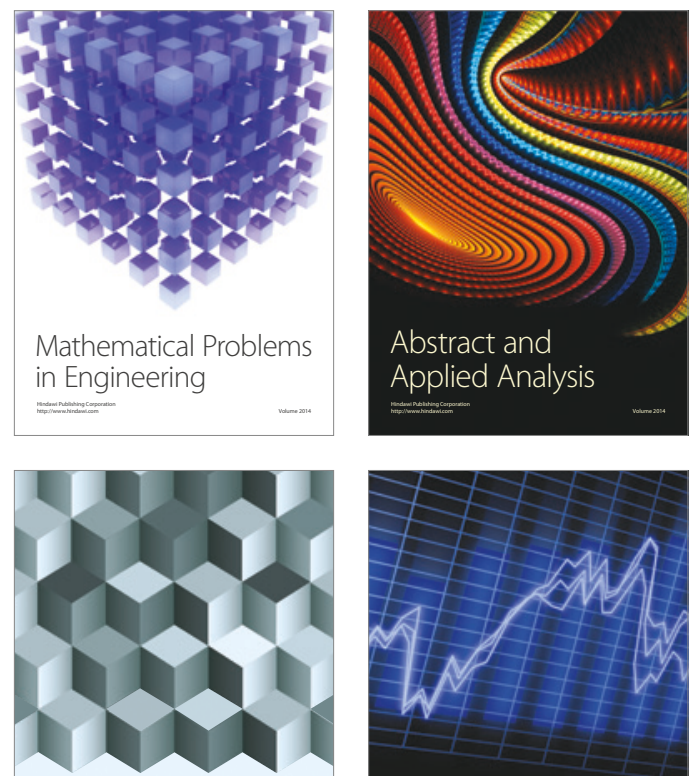

Journal of

Function Spaces

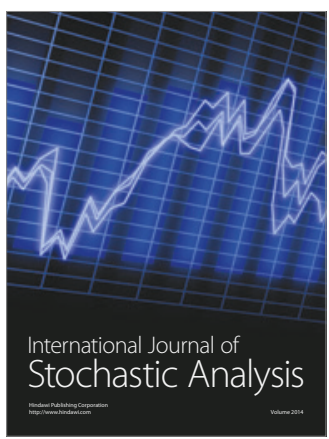

Probability and Statistics
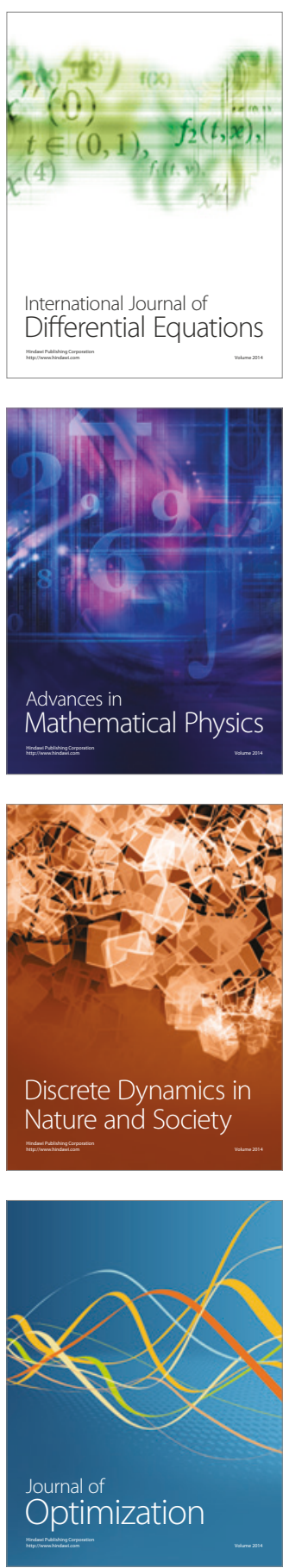